\title{
The Physical and Social Environment in Kaiser Permanente's Research Program on Genes, Environment and Health
}

Capturing Complexity: The Scientific, Societal, and Ethical Meanings of "Environment" in Genetic Research May 9, 2008 Stanford University

Catherine Schaefer, Ph.D. 


\section{Goal of the RPGEH}

To build a large and comprehensive resource for research on genetic and environmental influences on health and disease, by linking:

- Clinical data from Electronic Medical Records

- Participant survey data (over 400,000 KPNC members, to date)

- Environmental exposure data

- Genetic information on 500,000 consenting KPNC members 


\section{Aims of the RPGEH}

To enable KP and collaborating scientists to conduct research on genetic and environmental influences on disease susceptibility, course of disease, and response to treatment

To conduct research to translate findings into improvements in medical care and public health for KP members and public

Conduct research on the ethical, legal and social implications of genetic research and the use of genomic information in medical care. 


\section{Development of RPGEH}

Division of Research

Funded by private foundations and Kaiser Permanente

Inform Membership - understand expectations and concerns, engage interest in participation

Convene Community and Scientific Advisory Panels

Build RPGEH Database -- reorganize de-identified electronic clinical data into disease- condition databases

Survey Membership (1.9 million adults) -- to obtain demographic, environmental, and behavioral data

Develop Biorepository -- first 100,000 samples to be obtained in 2008- 2009 


\section{The E in RPGEH -- the Social and Physical Environment A critical factor in the program's success}

- Our Community Advisory Panel (CAP) has encouraged us to emphasize the environmental as well as genetic determinants of disease.

- Our recognition that all common diseases involve both environmental and genetic factors, interacting in complex patterns.

- Inclusion of environmental data sets RPGEH apart from many of the other biobank projects currently underway.

\section{Environment is broadly defined:}

- Host or personal environment

- Social environment

- Built environment

- Toxins / pollutants in the physical environment 


\section{Development of Environmental Data for RPGEH}

\section{Host or Personal Environment}

- Surveys include individual level data, e.g. marital status, education, income, occupation, stressful events, experience of discrimination, smoking, alcohol, physical activity, diet, etc.

- Biospecimens (blood, urine, saliva) are a source of data on individual level host factors with social implications (e.g., cortisol and other hormones) or other exposures (e.g., viral antibodies; PCBs)

- Biomonitoring of EMF or radiation exposure 


\section{Development of Environmental Data for RPGEH}

\section{Social and Built Environment}

- Area or group measures of social environment - US Census

- As local as a block group of 1,000 people

- Area databases on social factors and built environment; e.g., food and alcohol outlets, parks, locations of schools, highways, crime statistics

- "OurSpace" , a collaboration of DOR/KP, UCSF, and UCB, is creating an ecologic database of the social environment

- Building a GIS database on social factors and built environment including census data, food and alcohol outlets, parks, school locations, crime, etc.

- Database linkable to KP members through residence 


\section{Development of Environmental Data for RPGEH}

Physical Environment (Toxins and Pollutants)

- Collaboration with State environmental scientists to access CHAPIS \& air pollution data

- Pesticide and industrial exposures in air, soil, and water

- Additional toxic / host factors measured in biospecimens 


\section{Considerations in Relating Environment to Health}

\section{The effects of time}

- The environment changes over time - many databases are not longitudinal

- There is a lag between exposure and expression of disease

- Some environmental effects may depend on stage of development

\section{Levels of analysis}

- Beware reductionism -- Neighborhood Deprivation is not just an approximation of individual level SES data

- Avoid ecological fallacy - causal attribution to a social factor that is actually due to unmeasured confounder 


\section{ain: KAISER PERMANENTE。}

\section{Division of Research}

Key Personnel

Catherine Schaefer, Ph.D., Executive Director, RPGEH

Neil Risch, PhD, Co-Director, RPGEH

Joe Selby, MD, MPH, Director, Division of Research, KPNC

Sarah Rowell, MPH, Assoc Dir. Research Operations, RPGEH

Carol Somkin, PhD, ELSI Core

Elize Brown, JD, PhD, Community Outreach and Education Core Stephen Van Den Eeden, PhD, Environment Core

Charles Quesenberry, PhD, Study Design and Biostatistics Core

Barry Miller, PhD, Director of Research Admin., DOR

Larry Kushi, ScD, Assoc Director, DOR

Lisa Croen, PhD, Biorepository Design Core

Lisa Barcellos, PhD, Autoimmune Disease Registry 